\title{
OPEN Primary glia cells from bank vole propagate multiple rodent-adapted scrapie prions
}

\author{
Karla A. Schwenke ${ }^{1,2,3 凶}$, Joo-Hee Wälzlein ${ }^{1,3}$, Agnieszka Baver $^{2}$, Achim Thomzig $^{1}$ \& \\ Michael Beekes ${ }^{1}$
}

Since the beginning prion research has been largely dependent on animal models for deciphering the disease, drug development or prion detection and quantification. Thereby, ethical as well as cost and labour-saving aspects call for alternatives in vitro. Cell models can replace or at least complement animal studies, but their number is still limited and the application usually restricted to certain strains and host species due to often strong transmission barriers. Bank voles promise to be an exception as they or materials prepared from them are uniquely susceptible to prions from various species in vivo, in vitro and in cell-free applications. Here we present a mainly astrocyte-based primary glia cell assay from bank vole, which is infectible with scrapie strains from bank vole, mouse and hamster. Stable propagation of bank vole-adapted $\mathrm{RML}$, murine $22 \mathrm{~L}$ and $\mathrm{RML}$, and hamster $263 \mathrm{~K}$ scrapie is detectable from 20 or 30 days post exposure onwards. Thereby, the infected bank vole glia cells show similar or even faster prion propagation than likewise infected glia cells of the corresponding murine or hamster hosts. We propose that our bank vole glia cell assay could be a versatile tool for studying and comparing multiple prion strains with different species backgrounds combined in one cell assay.

Prion diseases are transmissible, fatal neurodegenerative disorders which include Creutzfeldt-Jakob disease (CJD) in humans, scrapie in sheep and goats, bovine spongiform encephalopathy (BSE) in cattle and chronic wasting disease (CWD) in cervids. They are caused by prions - proteinaceous infectious particles - which essentially consist of host-encoded, aggregated and self-propagating prion protein $(\mathrm{PrP})^{1}$. The pathology is marked by abnormal PrP deposits, astrogliosis, neuronal loss and vacuolation, which lead to severe brain dysfunction and inevitably death. Despite intense research in the past decades no treatment is available so far. According to the generally accepted "protein-only" hypothesis the underlying etiological event is the conversion of the physiological, cellular prion protein $\mathrm{PrP}^{\mathrm{C}}$ to its pathological isoform $\mathrm{PrP}^{\mathrm{Sc}}$, which is set-off due to (i) spontaneous misfolding and aggregation, (ii) inherited genetic reasons or (iii) $\mathrm{PrP}^{\mathrm{Sc}}$ acquired by infection. Once $\mathrm{PrP}^{\mathrm{Sc}}$ is present, prion propagation is an autocatalytic and self-sustaining process occurring mainly in cells with high $\operatorname{PrP}^{\mathrm{C}}$ expression like neurons and astrocytes ${ }^{2-4}$.

Before gradually identifying prions and unravelling their nature, prion research was mostly dependent on animal bioassays, but even nowadays they are the gold standard for $\operatorname{PrP}^{\mathrm{Sc}}$ detection and titre determination ${ }^{5,6}$. Refinement, reduction and replacement (" $3 R$ ") of animal studies is desirable due to ethical reasons but also towards less cost and labour-intensive methods, therefore alternatives to bioassays such as versatile cell culture models are needed. Already early in prion research a first persistently infected cell line from mouse was established ${ }^{7}$, later further cell models were introduced (for review see ${ }^{8}$ ). One successful example of a bioassay alternative is the standard scrapie cell assay based on subcloned neuroblastoma N2a cells, which was developed for titre determination of murine scrapie (22L and RML) reaching equal sensitivity while being significantly faster and cheaper ${ }^{9}$. However, this assay only works for mouse-adapted scrapie strains, which is a common problem of most cell culture models: being limited to certain host-adapted prions only ${ }^{8,10}$.

While neurons are extensively studied in prion disease, the role of other cell types remain to be further elucidated. Although prion accumulation leads to neuronal degeneration or death, also non-neuronal cells such as astrocytes are infected and potent propagators of prions in vivo but also in vitro ${ }^{11}$. Nonetheless, cell models mainly exist for neurons with few exceptions for primary mouse ${ }^{11-13}$ or hamster ${ }^{14}$ glia cells, human stem-cell derived astrocytes ${ }^{15}$ or recently the first infectible astrocyte cell line ${ }^{16}$. Due to transmission barriers hamster or

${ }^{1}$ Prion and Prionoid Research Unit, Centre for Biological Threats and Special Pathogens, Robert Koch Institute, 13353 Berlin, Germany. ${ }^{2}$ Present address: Department of Infectious Diseases, Robert Koch Institute, 13353 Berlin, Germany. ${ }^{3}$ These authors contributed equally: Karla A. Schwenke and Joo-Hee Wälzlein. ${ }^{\varpi}$ email: SchwenkeK@ rki.de 
murine cells are generally restricted to infection with species-adapted prion strains. Bank voles, however, were found to be universal acceptors for prions ${ }^{17}$ making them fast in vivo models for CJD ${ }^{18}$ as well as CWD ${ }^{19}$, but also broaden the application area of in vitro assays like real-time quaking induced conversion (RT-QuIC) ${ }^{20,21}$ and protein misfolding cyclic amplification (PMCA) ${ }^{22}$. So far only two cell lines expressing bank vole PrP were engineered being susceptible to murine scrapie (22L) and native $\mathrm{CWD}^{23}$, but no primary bank vole cell culture infectible with prions exists to the best of our knowledge. Cell lines have the advantage of providing fast and cheap cell assays, which are easy to maintain and have the potential for gene-editing. Benefits of primary compared to immortalised cells include a more natural simulation of an infection; for instance persistently prion-infected cell lines do not show cytotoxicity in contrast to prion-infected cells in vivo ${ }^{12,24}$. Also, in case of primary glia cells the post-mitotic state allows long-term cultivation without the need for passaging, which mimics the long incubation periods of prions and slow $\mathrm{PrP}^{\mathrm{Sc}}$ accumulation in vivo and further eliminates the risk of losing infectious titres due to disproportionally faster cell proliferation than prion propagation ${ }^{25}$. A primary bank vole cell assay infectible with prions could provide a tool for investigating a variety of prion strains from different host species under more natural conditions in a single cell model. In analogy to our already existing, robust hamster glia cell assay ${ }^{14,26}$, we aimed to establish a primary glia cell culture from bank vole, which would be susceptible to multiple prion strains from different species and exhibit stable prion propagation. Initially, a proof-of-concept with multiple rodent-adapted scrapie strains was to be accomplished, in order to provide a platform for the testing of more strains with different species background and native isolates such as CWD or CJD in the future. Here we present a primary mainly astrocyte-based cell assay from bank vole, which shows stable propagation of four scrapie strains from bank vole, mouse and hamster.

\section{Results}

Establishment of primary bank vole glia cell culture. The primary bank vole glia cell culture (BV-Glia) was established based on our protocol for Syrian hamster glia cells ${ }^{14,26}$ and the protocol for murine C57BL/6 glia cells (BL6-Glia) from the Kettenmann Lab (Max Delbrück Center, Berlin, Germany) with modifications specific for bank vole glia. The conventional protocol of isolating cells directly into serum-containing DMEM resulted in a very strong proliferation of bank vole cells, making them inapplicable for infection assays with required longterm cultivation without passaging. Changing the isolation protocol to a serum-free neurobasal medium supplemented with astrocytic-growth-promoting Bottenstein's G5 supplement first induced an intermediate state of 3D cell aggregate formation during the first days until cells differentiated and became adherent with a distinct fibrous morphology, which is similarly described in the literature ${ }^{27,28}$. Only after obtaining glia cells in a more mature state towards glial differentiation in the second passage, cells were used for infection assays and culture medium was switched to serum-containing DMEM to stabilise cells. The primary glia cell culture could then be maintained for at least 100 days without passaging, but an even longer cultivation period can be assumed, since we know from our primary transgenic mice glia culture that up to 150 days are possible ${ }^{13}$.

Characterisation of the glia cell culture. Since no specific astrocyte selection was performed, composition of the mixed glia cell culture was investigated by flow cytometry at the same passage and time point after plating cells analogue to prion inoculation. For characterisation the common astrocyte-specific cell markers glial fibrillary acidic protein (GFAP) and glutamate transporter 1 (GLT-1) were used. Additionally, expression of $\operatorname{PrP}^{\mathrm{C}}$ was investigated since this is known to be essential for successful prion propagation ${ }^{29,30}$. Flow cytometry analysis (Fig. 1) revealed a high content of astrocytes in the mixed culture with $94 \%$ in the double-positive GFAP+ GLT$1+$ cell population after gating for intact and single cells, of which more than $92 \%$ were GFAP+and almost $97 \%$ GLT-1+. These cells showed an overall high PrP expression on $>99 \%$ of the cells. Further consistence of the remaining negative cell population was not determined. A Western blot showing relative $\mathrm{PrP}^{\mathrm{C}}$ levels of BL6 and BV-Glia each obtained from a confluently grown 6-well interestingly revealed a higher $\mathrm{PrP}^{\mathrm{C}}$ content of BL6-Glia compared to the BV-Glia (supplementary files p. 5).

PrPSc propagation in the glia infection assay. BV-Glia were inoculated with rodent-adapted scrapie prions 22L and RML from C57BL/6 mice (BL6-22L, BL6-RML; $n=3$ ), bank vole-adapted RML (BV-RML; $n=3$ ) and $263 \mathrm{~K}$ from hamster $(\mathrm{n}=2)$. For each inoculum the corresponding, non-infectious brain homogenate $(\mathrm{NBH})$ was used as mock control. Cells were exposed to each inoculum ( $10 \mu \mathrm{l}$ of $1 \%$ brain homogenate) for 3 days, subsequently unattached material was removed and first samples were harvested as reference for residual inoculum (referred to as 3 days post exposure [dpe]). All four scrapie strains were efficiently propagated in BV-Glia, which was detected via Western blot (Fig. 2). Thereby, clearly increasing amounts of protease-resistant pathological PrP $\left(\mathrm{PrP}^{\mathrm{res}}\right)$ could be observed starting with first faint signals at 20 dpe and manifesting at 30 dpe onwards (BL6-22L, BL6-RML, BV-RML) or distinct signals already at the earliest time point at 20 dpe, respectively (263K). The increase of $\mathrm{PrP}^{\mathrm{res}}$ from the reference time point at 3 dpe to the following time points at 20 to 60 dpe indicates accumulating $\mathrm{PrP}^{\mathrm{Sc}}$ formation. For $263 \mathrm{~K}$ this could be confirmed by performing the blot with the antibody $3 \mathrm{~F} 4$ binding to hamster but not vole PrP, which detected residual hamster inoculum at 3 dpe, but no accumulating vole $\mathrm{PrP}^{\text {res }}$ at the later time points anymore (supplementary files p. 4). Mock-infected cells were always found to be negative for $\mathrm{PrP}^{\text {res }}$ in Western blot (not shown).

BL6-22L-infected BL6-Glia $(\mathrm{n}=3)$ showed a slightly faster and stronger prion propagation compared to the bank vole cells (distinct Western blot signals already at 20 dpe compared to 30 dpe, Fig. 2A). In contrast, inoculation with BL6-RML $(n=3)$ revealed a less efficient prion propagation in BL6-Glia, for which in only one of three experiments a very faint signal could be seen at 60 dpe (Fig. 2B). This indicates infectibility in principle but the need for a prolonged cultivation time. Surprisingly, other than the murine cells $\mathrm{PrP}^{\mathrm{res}}$ signals in 
A

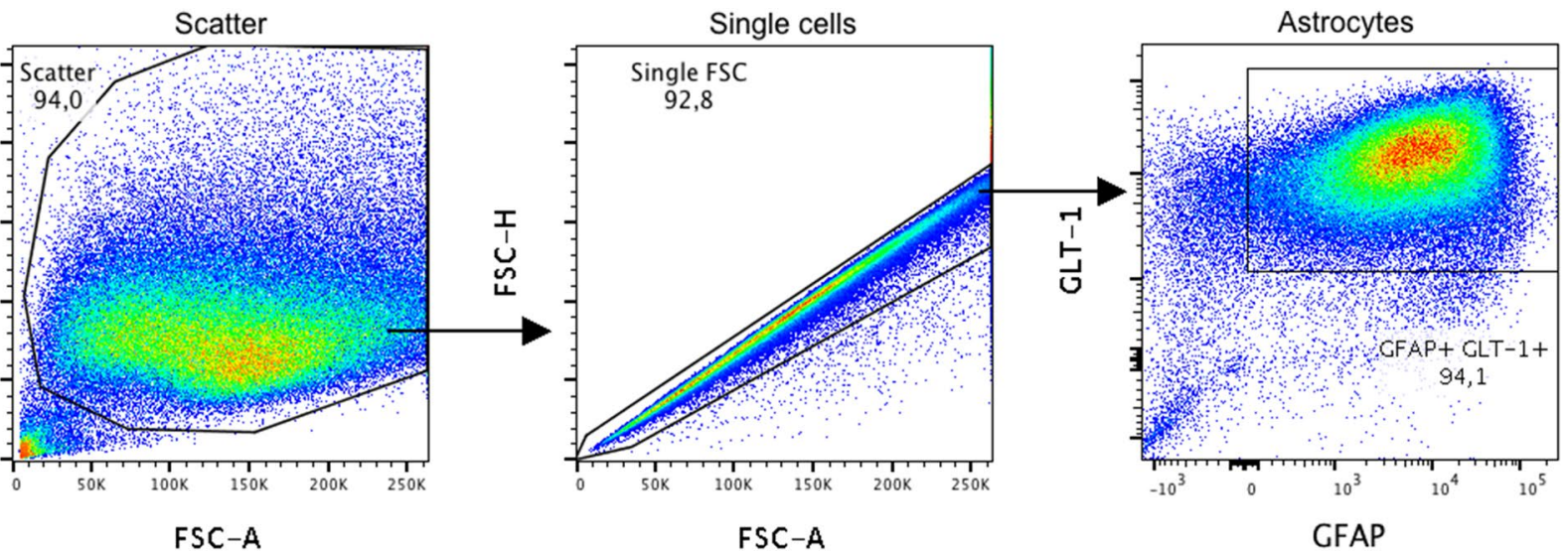

B
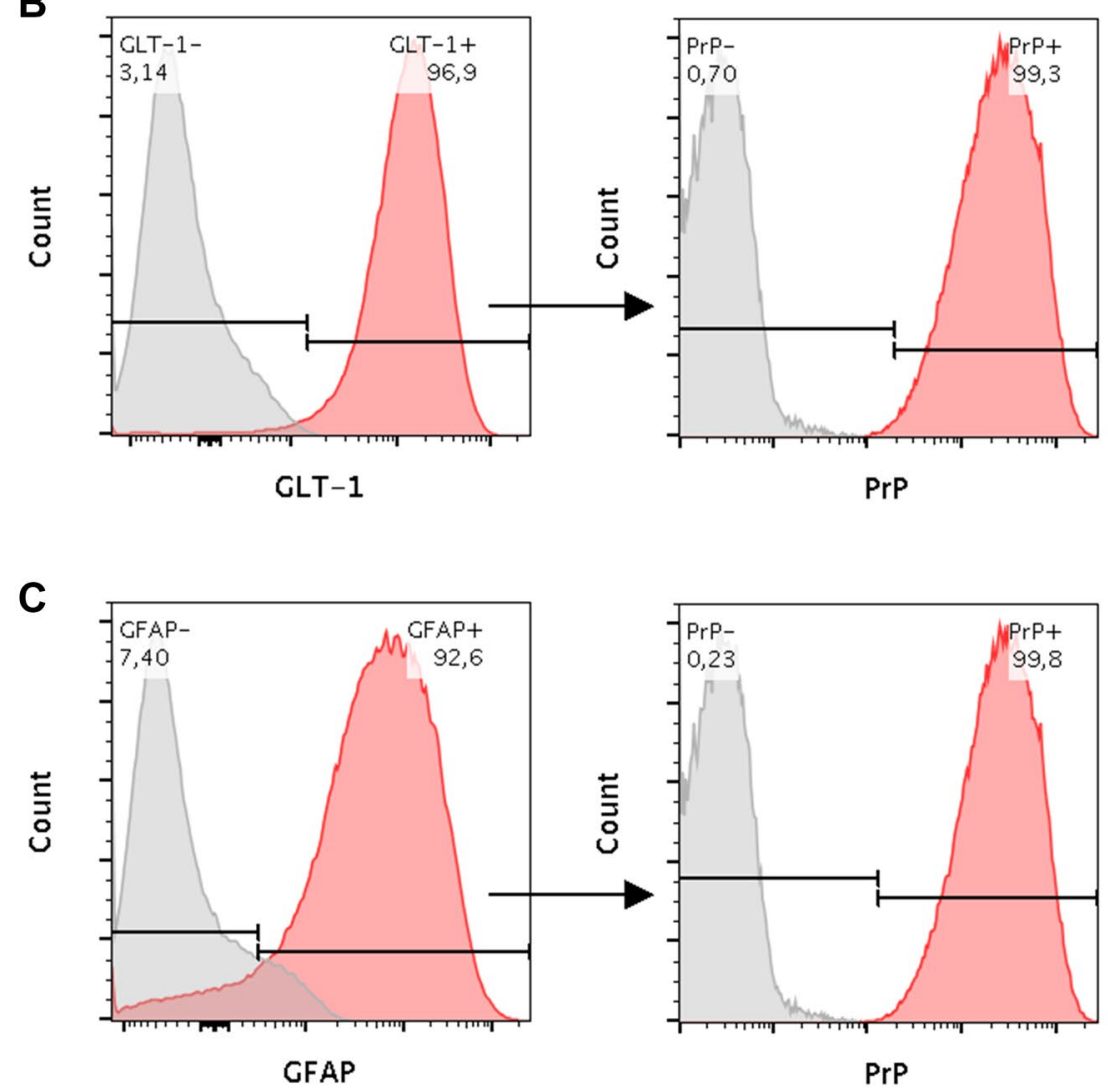

Figure 1. Composition of primary bank vole glia cells at the time of infection ( 20 days post isolation, passage 2). Characterisation was performed by flow cytometry analysis based on expression of GFAP and GLT-1, two specific markers for cells of astrocytic phenotype. Additionally, levels of $\operatorname{PrP}^{\mathrm{C}}$ expression were determined as crucial factor for prion propagation. (A) Proportion of the analysed cells, which were found to co-express GFAP and GLT-1. Cell debris and doublets were excluded from the analysis by gating as illustrated. (B) Histogram of those cells, which were stained for GLT-1 and (C) for GFAP. $\operatorname{PrP}^{\mathrm{C}}$ expression of each subpopulation is shown in the respective second panel. Red areas of the histograms refer to stained samples and grey areas to the corresponding control incubated without the target antibody. The experiment was carried out in triplicate, of which one representative is shown. According to the analysis the glia cell culture mainly consisted of astrocytes with high $\mathrm{PrP}^{\mathrm{C}}$ expression, which provided the basis for an infectible cell assay. FSC: forward scatter, SSC: side scatter.

BL6-RML-inoculated BV-Glia were detectable already from 30 dpe onwards (Fig. 2D), comparable to BL6-22L or BV-RML prions in bank vole cells.

Also, inoculation of BV-Glia with hamster $263 \mathrm{~K}$ revealed an efficient prion propagation with strong signals as early as 20 dpe already reaching maximal propagation only 10 days later, which is on the same level as known from the currently established version of our hamster glia cell assay (unpublished data). 


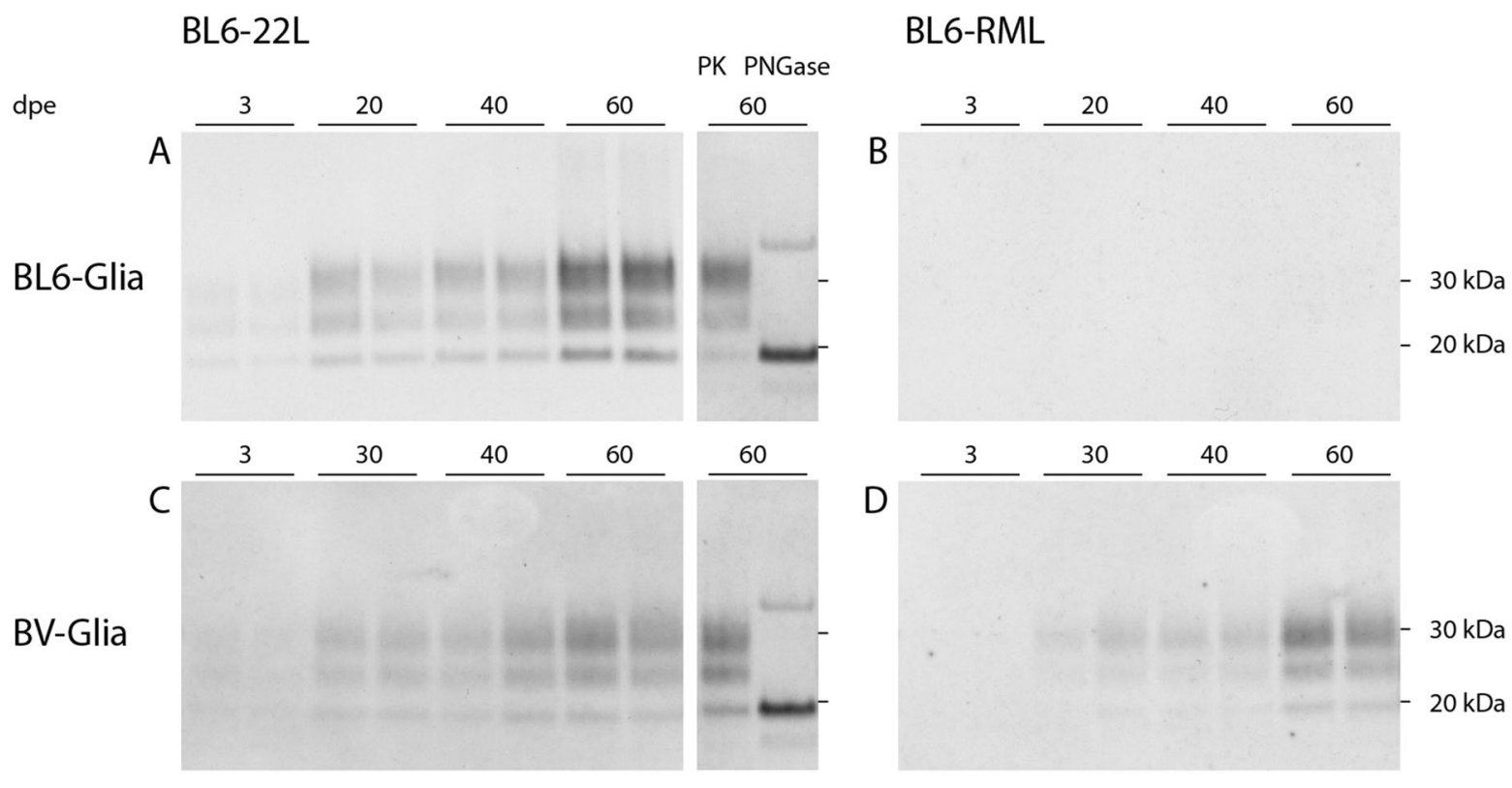

BV-RML

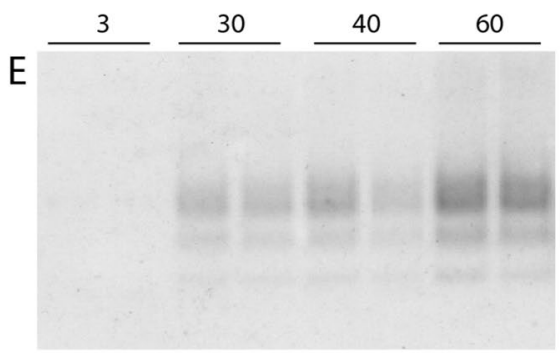

PK PNGase

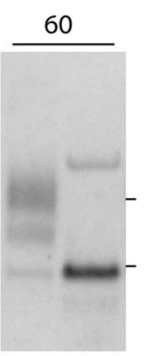

\section{Hamster 263K}

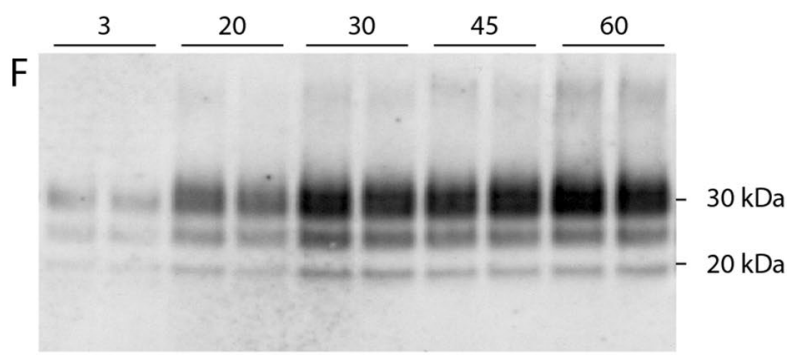

Figure 2. Western blot analyses of PK-digested cell lysates of prion-infected glia cells from C57BL/6 mice and bank vole. Glia cells from mouse $(\mathbf{A}, \mathbf{B})$ or bank vole $(\mathbf{C}-\mathbf{F})$ were exposed to scrapie prion strains: mouseadapted 22L (A, C), mouse-adapted RML (B, D), bank vole-adapted RML (E) and hamster-adapted 263K (F) for three days and harvested at the indicated time points. The immunoblots illustrate the propagation of pathological prion protein in cells over the cultivation time dependent on the seeding material. All four strains show an efficient propagation in bank vole glia cells, even for BL6-RML which shows no sign of infection in mouse cells up to 60 dpe. In A, C and E 60 dpe-samples deglycosylated with peptide-N-glycosidase F (PNGase F) are shown with a clear shift of the di- and monoglycosylated band to the unglycosylated band at $19 \mathrm{kDa}$. The band at $\sim 35 \mathrm{kDa}$ is considered an artefact of the deglycosylation process itself ${ }^{46}$. Representative Western blots of infection assays are shown, which were stained with anti-PrP antibody ICSM-18 (1:4000) ( $n=3$, except: $263 \mathrm{~K}: \mathrm{n}=2$; all in duplicates). The corresponding full-length Western blots are provided in the supplementary files p. 7-9 and exemplary for BV-RML blots of the three replicate experiments are shown on p. 6.

In contrast, mouse and bank vole-adapted ME7 scrapie brain homogenates were tested twice under the same conditions but did not show any sign of propagation up to 60 dpe in neither BL6 nor BV-Glia. To exclude that the absence of $\mathrm{PrP}^{\mathrm{res}}$ signals for some strains is caused by higher proteinase $\mathrm{K}(\mathrm{PK})$ sensitivity, all 60 dpe-cell lysates were additionally digested with lower PK concentrations. However, these still did not reveal any signals in the blank lanes, but only resulted in incomplete digest of accumulated PrP for the other signals (supplementary files p. 2).

In order to verify the Western blot signals as $\mathrm{PrP}^{\text {res }}$, deglycosylation was carried out with the N-glycosidase PNGase F. For the representatively tested cell lysates (cells infected with BL6-22L and BV-RML at 60 dpe) a uniform shift of the di- and monoglycosylated bands to the unglycosylated band at approximately $19 \mathrm{kDa}$ could be observed as expected (Fig. 2A,C,E), which confirms the identity of the immunolabelled prion protein. Interestingly, strain characteristics of $\mathrm{PrP}^{\mathrm{Sc}}$ in terms of glycosylation pattern were not retained when propagated by the glia cells. For all strains showing a productive infection a dominant diglycosylated band was observed. 


\section{Discussion}

A mainly astrocytic primary bank vole glia assay suitable for long-term cultivation. To the best of our knowledge this is the first report of a primary glia cell culture from bank vole, which shows a stable propagation of rodent-adapted scrapie prions of murine, hamster and bank vole origin. The primary mixed glia cell culture revealed a high content of astrocytic cells in flow cytometry, of which $94 \%$ belonged to the doublepositive cell population for the specific astrocyte markers GFAP and GLT-1. While GFAP has been seen as a definite single astrocyte marker for a long time, nowadays this assumption is revisited and the use of at least two markers recommended ${ }^{31}$. We included GLT-1 in our analysis, which is a potent marker for cells with astrocytic phenotype $^{32}$ and the predominant glutamate transporter in the developed brain ${ }^{33,34}$. Interestingly, in a first flow cytometry analysis for test purposes a tremendous increase of GLT-1+cells was observed between the initially applied protocol with serum-containing medium and the subsequently adapted bank vole protocol with serumfree cultivation for cell isolation (not shown), which points to more mature astrocytes in the cell mix of the latter. Previous studies ${ }^{35,36}$ have shown that exposing freshly isolated glia cells to serum changes their expression profile and apparently keeps them in an immature state as opposed to serum-free cultivation. In contrast, a later cultivation in serum does not seem to alter cell composition anymore. This would explain the impeded proliferation of the bank vole glia cells and the quicker shift to a post-mitotic state when the isolation protocol was changed. It remains unclear why absence of serum does not appear to be crucial for mouse glia cells, but possibly efficiency of the BL6-Glia infection assay could be further enhanced by switching to serum-free cell isolation. Surprisingly, the relative $\mathrm{PrP}^{\mathrm{C}}$ level of the BL6-Glia still seems to be higher than of the BV-Glia. The combination of the serum-free medium with the astrocytic-growth-promoting Bottenstein's G5 supplement likely contributes to the relatively pure astrocyte culture with little contamination of other cells like microglia or oligodendrocytes, which are not considered a site of pronounced prion propagation ${ }^{37-39}$. Hence, this isolation protocol seems to promote the differentiation of the cells to a more mature astrocyte cell type, which seems to favour prion propagation.

Equal or faster propagation rate of scrapie prions in bank vole glia compared to murine cells. In this bank vole glia cell assay all four scrapie strains led to a productive infection and $\operatorname{PrP}^{\mathrm{Sc}}$ accumulation within relatively short cultivation periods with immunoblot signals being first visible at 20 or latest 30 dpe. Thereby, the murine strains showed an almost similar fast propagation (BL6-22L) as or even an accelerated one (BL6-RML) within half of the cultivation time than in likewise infected BL6-Glia. Even for the non-murine 263K hamster scrapie strain the propagation rate was as fast as in hamster glia cell assays carried out in our laboratory. This points to a broader and increased susceptibility of prion strains in bank vole cells as already reported in vivo and in vitro and suggests a highly versatile usability of this glia cell-based assay.

The ME7 scrapie strain is known to be slower in propagation in vivo and in cell culture, and to show a different tropism than e.g. $22 \mathrm{~L}$ or RML ${ }^{24,40-42}$. Since ME7 was found to propagate in neurons, it seems possible that this mainly astrocyte-based assay is refractory to ME7 infection in general or at least needs substantially prolonged cultivation periods. However, lower titres of the used ME7 brain homogenates or higher sensitivity to PK seems unlikely to be causative when compared to the other strains (supplementary files p. 1,2). A complementary neuronal cell assay from bank vole could possibly provide more insights into the tropism of ME7.

For prion-infected cell lines analyses of $\operatorname{PrP}^{\mathrm{sc}}$ formation is generally carried out after (serial) passaging of cells to exclude residual inoculum. Since no cell passaging was performed after infecting our primary cells, we consider the clear increase of $\mathrm{PrP}^{\text {res }}$ over time in Western blot as evidence for biological $\mathrm{PrP}^{\mathrm{Sc}}$ amplification in our bank vole glia cells, as it could be confirmed for infection with $263 \mathrm{~K}$ by an alternative antibody staining.

Bank vole as universal prion acceptor in a cell model. Our results confirm the assumption that bank vole is an exceptional acceptor for prions in cell culture as well. Remarkably, by infecting bank vole glia not only the transmission barrier is overcome, but partly also the cultivation time even shorter than for murine prions in mouse cells. Therefore, this assay is a promising versatile tool with the potential for a single, universal platform, which could combine studies with several prion strains from different host species in one assay and thus allow comparative analysis. Prion research still lacks in such a universal cell model and also propagation of human CJD prions in cell culture remains challenging to date. Apart from stem cell-derived astrocyte or cerebral organoid cultures ${ }^{15,43}$, recently, the first primary astrocyte-based cell assay propagating MM2 and VCJD but not MM1 prions was reported by Wälzlein et al. ${ }^{13}$. The analogue set-up of this astrocyte-based assay combined with the exceptional susceptibility of bank voles to various prion strains might allow successful infection of our bank vole glia with CJD prions as well. In particular, propagation of MM1 prions might be possible based on in vivo studies, in which MM1 isolates caused the shortest survival time in bank voles among CJD strains ${ }^{17,18}$. Furthermore, our cell assay is cheaper, easier and less labour-intensive than stem-cell-derived astrocyte or organoid cultures, and notably bioassays. Beyond the aim of implementing the 3R principles for the sake of animal protection in general, bank voles demand particular attention in this direction since they are unusually prone to stress and require extremely careful handling ${ }^{44}$. Replacing animal experiments with the use of bank vole-tissue for in vitro studies would therefore represent a highly desirable step forward in terms of animal welfare.

\section{Materials and methods}

Ethics statement. All applicable international, national, and institutional guidelines for the care and use of animals were followed. The study was conducted in strict accordance with the European Directive on the Protection of Animals used for Scientific Purposes (2010/63/EU), the German Animal Welfare Act (TierSchG), and the Regulation on the Protection of Animals used for Experimental and other Scientific Purposes (TierSchVersV). Euthanasia of animals for scientific purposes was approved by and reported to the institutional and local 
governmental authorities (State Office of Health and Social Affairs - Landesamt für Gesundheit und Soziales Berlin, Germany; approval IDs: T 0300/15, TN 0001/21 and T 0256/15).

Animals and tissues. Neonatal C57BL/6 mice and bank voles (Myodes glareolus, PrP genotype Bv109M ${ }^{19}$ ) were obtained from colonies in the animal facility of Robert Koch Institute (RKI). Initial breeding pairs of bank voles were received from Istituto Superiore di Sanità, Rome, Italy.

Brain tissue of terminally ill hamsters with $263 \mathrm{~K}$ were taken from stock at RKI. Murine 22L, RML and ME7 as well as bank-vole RML and ME7 brain tissue samples were kindly provided by the Gilch and Schätzl Lab, University of Calgary, Canada.

Glia cell isolation and cultivation. Primary glia cells were isolated from brains of neonatal bank voles (0-3 days post natum, euthanasia by decapitation) based on Buonfiglioli et al. ${ }^{45}$ with modifications. Briefly, the cerebellum, olfactory bulb and meninges were removed and the brains washed three times with ice-cold Hank's Balanced Salt Solution (HBSS). Enzymatic dissociation was carried out by incubating brain tissue with $10 \mathrm{mg} /$ $\mathrm{ml}$ Trypsin (Biochrom, Germany) and $0.5 \mathrm{mg} / \mathrm{ml}$ DNase I (Roche, Switzerland) in HBSS for two minutes at first and subsequently with $5 \mathrm{mg} / \mathrm{ml}$ DNase I in HBSS while gently triturating by pipetting. The tissue suspension was centrifuged at $120 \times \mathrm{g}$ for $10 \mathrm{~min}$, cells resuspended in neurobasal medium containing $1 \%$ G5 supplement, $1 \%$ GlutaMAX and $100 \mathrm{U} / \mathrm{ml}$ Penicillin/Streptomycin (all Gibco ${ }^{\mathrm{Tm}}$ Thermo Fisher, USA; referred to as NBM complete) and quickly plated on poly-L-lysine (PLL; $0.01 \%[\mathrm{w} / \mathrm{v}]$ )-coated T75 flasks, so that 2.5 brains were allocated per flask. Cells were maintained under humid conditions with $5 \% \mathrm{CO}_{2}$ at $37^{\circ} \mathrm{C}$. After 2 days some adherent cells but mainly semi-adherent $3 \mathrm{D}$ cell aggregates could be observed. In order to reduce cytotoxic cell debris and tissue chunks, cells were washed once very gently with phosphate-buffered saline (PBS) and incubated for further 2 days. During this time most cells became adherent and could be washed thoroughly for removal of remaining cell debris. After another 2-3 days cells have grown confluent and were split in a ratio of 1:3. After further 7 days (14 days post isolation) cells were ready to be harvested and either directly seeded for experiments or cryopreserved for later use.

Glia cells from neonatal C57BL/6 mice were isolated likewise, except that Dulbecco's Modified Eagle's Medium (DMEM) containing 10\% foetal bovine serum (FBS; Pan Biotech, Germany, P30-3031), 1\% GlutaMAX and $100 \mathrm{U} / \mathrm{ml}$ Penicillin/Streptomycin was used instead of NBM complete. Due to the presence of serum cells were adherent after 2 days post isolation already, could be washed thoroughly for four times and were cultivated for another 5 days until cells have grown confluent. Seven days post isolation mouse glia cells were harvested and used for experiments without further passaging.

Flow cytometry. For determining the cellular composition of the bank vole glia culture at the time of infection, expression of the astrocyte-specific markers glial fibrillary acidic protein (GFAP) and glutamate transporter 1 (GLT-1) was investigated as well as PrP expression. Samples were analysed in triplicates and the corresponding Fluorescence Minus One (FMO) controls in duplicates. Furthermore, unstained and compensation controls were performed for necessary adjustments of the laser channels and compensation of fluorophores. Briefly, glia cells of a confluent T75 flask were detached with Accutase, centrifuged, resuspended in FACS buffer (PBS containing $2 \%[\mathrm{v} / \mathrm{v}]$ FBS and $0.1 \%[\mathrm{w} / \mathrm{v}]$ sodium azide) and equally distributed on a round-bottom 96-well plate. Prior to intracellular staining cells were fixed with $2 \%(\mathrm{w} / \mathrm{v})$ paraformaldehyde, cell membranes permeabilised with $0.5 \%(\mathrm{w} / \mathrm{v})$ saponin and unspecific binding sites blocked with $1 \%(\mathrm{w} / \mathrm{v})$ bovine serum albumin. Cells were then incubated for $30 \mathrm{~min}$ on ice with previously titrated dilutions of primary antibodies recombinant anti-GFAP PE-conjugated [REA335, Miltenyi Biotec, Germany]: 1:100; polyclonal rabbit anti-GLT-1 [ab41621, Abcam, UK]: 1:200; monoclonal mouse anti-PrP [SAF84, Bertin, France]: 1:400) and subsequently, after washing once with FACS buffer, for $15 \mathrm{~min}$ at room temperature with the secondary antibodies (Alexa488-conjugated anti-rabbit IgG and Alexa647-conjugated anti-mouse IgG: 1:500 [Thermo Fisher, USA]). Afterwards cells were washed twice and finally resuspended in $200 \mu \mathrm{l}$ FACS buffer. $150 \mu \mathrm{l}$ of each sample was analysed using the MACSQuant Analyzer 10 (Miltenyi Biotec, Germany) and the data evaluated using FlowJo 10 software (BD Life Science, USA). Cell debris and doublet cells were excluded based on scatter signals.

Cell-based infection assay. For the infection assay medium for mouse glia cells was changed from DMEM to DMEM/F12 with the same supplements for better cell viability during long-term cultivation, and for bank vole cells NBM complete was switched to DMEM complete supplemented with $1 \% \mathrm{~N}-2$ supplement $\left(\mathrm{Gibco}^{\mathrm{Tm}}\right.$ Thermo Fisher, USA). Mouse or bank vole glia cells were plated at $1 \times 10^{5}$ or $5 \times 10^{4}$ cells/well, respectively, in PLL-coated 6-well plates and grown semi-confluent for 5 days. Prior inoculation $10 \%$ brain homogenates were diluted 1:10 in the respective cell culture medium and sonicated for $30 \mathrm{~s}$ at $300 \mathrm{~W}$ in a microplate horn sonicator (Misonix 3000, USA). Cells were exposed to $10 \mu \mathrm{l}$ of the respective seeds in $2 \mathrm{ml} \mathrm{medium} /$ well for 3 days, subsequently washed once with PBS for removal of non-incorporated seeds and supplemented with $3 \mathrm{ml}$ fresh medium. Cells were cultivated until the indicated time points with a weekly medium change. For all experiments one sample was taken at 3 dpe as a reference for initial seeds, further cells were harvested at 20, 30, 40, 45 or $60 \mathrm{dpe}$ as stated by lysing cells with $100 \mu \mathrm{l} /$ well of $1 \%$ sarcosyl.

SDS-PAGE and Western blot. Cell lysates in $1 \%$ sarcosyl were digested with $75 \mu \mathrm{g} / \mathrm{ml}$ PK (Roche, Switzerland) for $1 \mathrm{~h}$ at $37^{\circ} \mathrm{C}$ and subsequently denatured in an equal volume of $2 \mathrm{x}$ sample loading buffer at $110^{\circ} \mathrm{C}$. Deglycosylation was performed with peptide- $N$-glycosidase F (PNGase F; New England Biolabs, USA) following the manufacturer's instructions ${ }^{46}$. For this $15 \mu \mathrm{l} \mathrm{PK}$-digested and denatured samples were mixed with $2 \mu \mathrm{l}$ each of 10x GlycoBuffer2, NP-40 and PNGase F and incubated for $2 \mathrm{~h}$ at $37^{\circ} \mathrm{C} .20 \mu \mathrm{l}$ of either type of samples were 
run on BOLT 4-12\% Bis-Tris mini protein gels for SDS-PAGE and electroblotted using the iBlot 2 dry blotting system (Thermo Fisher, USA). Polyvinylidene fluoride membranes were probed with anti-PrP monoclonal antibody ICSM-18 (1:4000; D-Gen, UK) or 3F4 (1:2000; inhouse production) overnight and anti-mouse IgG alkaline phosphatase-linked secondary antibody (1:5000; Dako, USA). Membranes were incubated with CDPStar chemiluminescent substrate (Thermo Fisher, USA) for alkaline phosphatase chemiluminescence reaction and detected on Amersham Hyperfilm ${ }^{\mathrm{ma}}$ ECL films (GE Healthcare, USA). Images were created in Illustrator 2021 (Adobe, USA).

\section{Data availability}

The data produced in these experiments are available from the corresponding author on request.

Received: 21 September 2021; Accepted: 25 January 2022

Published online: 09 February 2022

\section{References}

1. Prusiner, S. B. Novel proteinaceous infectious particles cause scrapie. Science (New York, N.Y.) 216, 136-144. https://doi.org/10. 1126/science.6801762 (1982).

2. Prusiner, S. B. Prions. Proc. Natl. Acad. Sci. U.S.A. 95, 13363-13383. https://doi.org/10.1073/pnas.95.23.13363 (1998).

3. Aguzzi, A. \& Calella, A. M. Prions: Protein aggregation and infectious diseases. Physiol. Rev. 89, 1105-1152. https://doi.org/10. 1152/physrev.00006.2009 (2009).

4. Watts, J. C., Balachandran, A. \& Westaway, D. The expanding universe of prion diseases. PLoS Pathog. 2, e26. https://doi.org/10. 1371/journal.ppat.0020026 (2006).

5. Brandner, S. \& Jaunmuktane, Z. Prion disease: Experimental models and reality. Acta Neuropathol. 133, 197-222. https://doi.org/ 10.1007/s00401-017-1670-5 (2017).

6. Watts, J. C. \& Prusiner, S. B. Mouse models for studying the formation and propagation of prions ${ }^{\star}$. J. Biol. Chem. 289, 19841-19849. https://doi.org/10.1074/jbc.R114.550707 (2014).

7. Clarke, M. C. \& Haig, D. A. Multiplication of scrapie agent in cell culture. Res. Vet. Sci. 11, 500-501. https://doi.org/10.1016/ S0034-5288(18)34291-7 (1970).

8. Pineau, H. \& Sim, V. L. From cell culture to organoids-model systems for investigating prion strain characteristics. Biomolecules 11, $106(2021)$.

9. Klöhn, P. C., Stoltze, L., Flechsig, E., Enari, M. \& Weissmann, C. A quantitative, highly sensitive cell-based infectivity assay for mouse scrapie prions. Proc. Natl. Acad. Sci. 100, 11666. https://doi.org/10.1073/pnas.1834432100 (2003).

10. Krance, S. H. et al. Cellular models for discovering prion disease therapeutics: Progress and challenges. J. Neurochem. 153, 150-172. https://doi.org/10.1111/jnc.14956 (2020).

11. Victoria, G. S., Arkhipenko, A., Zhu, S., Syan, S. \& Zurzolo, C. Astrocyte-to-neuron intercellular prion transfer is mediated by cell-cell contact. Sci. Rep. 6, 20762. https://doi.org/10.1038/srep20762 (2016).

12. Cronier, S., Laude, H. \& Peyrin, J. M. Prions can infect primary cultured neurons and astrocytes and promote neuronal cell death. Proc. Natl. Acad. Sci. U.S.A. 101, 12271-12276. https://doi.org/10.1073/pnas.0402725101 (2004).

13. Wälzlein, J.-H., Schwenke, K. A. \& Beekes, M. Propagation of cjd prions in primary murine glia cells expressing human prpc. Pathogens 10, 1060 (2021).

14. Pritzkow, S. et al. Quantitative detection and biological propagation of scrapie seeding activity in vitro facilitate use of prions as model pathogens for disinfection. PLoS One 6, e20384. https://doi.org/10.1371/journal.pone.0020384 (2011).

15. Krejciova, Z. et al. Human stem cell-derived astrocytes replicate human prions in a prnp genotype-dependent manner. J. Exp. Med. 214, 3481-3495. https://doi.org/10.1084/jem.20161547 (2017).

16. Tahir, W. et al. An astrocyte cell line that differentially propagates murine prions. J. Biol. Chem. 295, 11572-11583. https://doi.org/ 10.1074/jbc.RA120.012596 (2020).

17. Watts, J. C. et al. Evidence that bank vole prp is a universal acceptor for prions. PLoS Pathog. 10, e1003990-e1003990. https://doi. org/10.1371/journal.ppat.1003990 (2014).

18. Nonno, R. et al. Efficient transmission and characterization of creutzfeldt-jakob disease strains in bank voles. PLoS Pathog. 2 , e12. https://doi.org/10.1371/journal.ppat.0020012 (2006).

19. Di Bari, M. A. et al. Chronic wasting disease in bank voles: Characterisation of the shortest incubation time model for prion diseases. PLoS Pathog. 9, e1003219. https://doi.org/10.1371/journal.ppat.1003219 (2013).

20. Orrú, C. D. et al. Bank vole prion protein as an apparently universal substrate for rt-quic-based detection and discrimination of prion strains. PLoS Pathog. 11, e1004983. https://doi.org/10.1371/journal.ppat.1004983 (2015).

21. Mok, T. H. et al. Bank vole prion protein extends the use of rt-quic assays to detect prions in a range of inherited prion diseases. Sci. Rep. 11, 5231. https://doi.org/10.1038/s41598-021-84527-9 (2021).

22. Bélondrade, M. et al. Sensitive protein misfolding cyclic amplification of sporadic creutzfeldt-jakob disease prions is strongly seed and substrate dependent. Sci. Rep. 11, 4058. https://doi.org/10.1038/s41598-021-83630-1 (2021).

23. Walia, R., Ho, C. C., Lee, C., Gilch, S. \& Schatzl, H. M. Gene-edited murine cell lines for propagation of chronic wasting disease prions. Sci. Rep. 9, 11151. https://doi.org/10.1038/s41598-019-47629-z (2019).

24. Hannaoui, S. et al. Prion propagation and toxicity occur $<\mathrm{em}>$ in vitro $</ \mathrm{em}>$ with two-phase kinetics specific to strain and neuronal type. J. Virol. 87, 2535-2548. https://doi.org/10.1128/jvi.03082-12 (2013).

25. Ghaemmaghami, S. et al. Cell division modulates prion accumulation in cultured cells. Proc. Natl. Acad. Sci. 104, 17971-17976. https://doi.org/10.1073/pnas.0708372104 (2007).

26. Boerner, S., Wagenfuhr, K., Daus, M. L., Thomzig, A. \& Beekes, M. Towards further reduction and replacement of animal bioassays in prion research by cell and protein misfolding cyclic amplification assays. Lab. Anim. 47, 106-115. https://doi.org/10.1177/ $0023677213476856(2013)$

27. Vanhoutte, N., de Hemptinne, I., Vermeiren, C., Maloteaux, J. M. \& Hermans, E. In vitro differentiated neural stem cells express functional glial glutamate transporters. Neurosci. Lett. 370, 230-235. https://doi.org/10.1016/j.neulet.2004.08.039 (2004).

28. Michler-Stuke, A., Wolff, J. R. \& Bottenstein, J. E. Factors influencing astrocyte growth and development in defined media. Int. J. Dev. Neurosci. 2, 575-584. https://doi.org/10.1016/0736-5748(84)90035-2 (1984).

29. Weissmann, C., Büeler, H., Fischer, M., Sauer, A. \& Aguet, M. Susceptibility to scrapie in mice is dependent on prpc. Philos. Trans. Royal Soc. Lond. Ser. B, Biol. Sci. 343, 431-433. https://doi.org/10.1098/rstb.1994.0040 (1994).

30. Manson, J. C., Clarke, A. R., McBride, P. A., McConnell, I. \& Hope, J. Prp gene dosage determines the timing but not the final intensity or distribution of lesions in scrapie pathology. Neurodegener: J. Neurodegener. Disord., Neuroprot., Neuroregener. 3, 331-340 (1994).

31. Guttenplan, K. A. \& Liddelow, S. A. Astrocytes and microglia: Models and tools. J. Exp. Med. 216, 71-83. https://doi.org/10.1084/ jem.20180200 (2018). 
32. DeSilva, T. M., Borenstein, N. S., Volpe, J. J., Kinney, H. C. \& Rosenberg, P. A. Expression of eaat2 in neurons and protoplasmic astrocytes during human cortical development. J. Comp. Neurol. 520, 3912-3932. https://doi.org/10.1002/cne.23130 (2012).

33. Ullensvang, K., Lehre, K. P., Storm-Mathisen, J. \& Danbolt, N. C. Differential developmental expression of the two rat brain glutamate transporter proteins glast and glt. Eur. J. Neurosci. 9, 1646-1655. https://doi.org/10.1111/j.1460-9568.1997.tb01522.x (1997).

34. Regan, M. R. et al. Variations in promoter activity reveal a differential expression and physiology of glutamate transporters by glia in the developing and mature cns. J. Neurosci. 27, 6607-6619. https://doi.org/10.1523/jneurosci.0790-07.2007 (2007).

35. Foo, L. C. et al. Development of a method for the purification and culture of rodent astrocytes. Neuron 71, 799-811. https://doi. org/10.1016/j.neuron.2011.07.022 (2011).

36. Zamanian, J. L. et al. Genomic analysis of reactive astrogliosis. J. Neurosci. 32, 6391-6410. https://doi.org/10.1523/jneurosci.622111.2012 (2012)

37. Prinz, M. et al. Intrinsic resistance of oligodendrocytes to prion infection. J. Neurosci.: Off. J. Soc. Neurosci. 24, 5974-5981. https:// doi.org/10.1523/jneurosci.0122-04.2004 (2004).

38. Aguzzi, A. \& Zhu, C. Microglia in prion diseases. J. Clin. Investig. 127, 3230-3239. https://doi.org/10.1172/jci90605 (2017).

39. Carroll, J. A. \& Chesebro, B. Neuroinflammation, microglia, and cell-association during prion disease. Viruses https://doi.org/10. 3390/v11010065 (2019).

40. Carroll, J. A. et al. Prion strain differences in accumulation of prpsc on neurons and glia are associated with similar expression profiles of neuroinflammatory genes: Comparison of three prion strains. PLoS Pathog. 12, e1005551. https://doi.org/10.1371/journ al.ppat.1005551 (2016).

41. Sisó, S. et al. Disease phenotype in sheep after infection with cloned murine scrapie strains. Prion 6, 174-183. https://doi.org/10. 4161/pri.18990 (2012).

42. van Keulen, L. J. et al. Tse strain differentiation in mice by immunohistochemical prp(sc) profiles and triplex western blot. Neuropathol. Appl. Neurobiol. 41, 756-779. https://doi.org/10.1111/nan.12181 (2015).

43. Groveman, B. R. et al. Sporadic creutzfeldt-jakob disease prion infection of human cerebral organoids. Acta Neuropathol. Commun. 7, 90. https://doi.org/10.1186/s40478-019-0742-2 (2019).

44. Paul, L., Kirsch, P., Thomzig, A., Thöne-Reineke, C. \& Beekes, M. Practical approaches for refinement and reduction of animal experiments with bank voles in prion research. Berl. Münchener Tierärztliche Wochenschr. 131, 359-367 (2018).

45. Buonfiglioli, A. et al. <em $>$ let-7</em $>$ micrornas regulate microglial function and suppress glioma growth through toll-like receptor 7. Cell Rep. 29, 3460-3471.e3467. https://doi.org/10.1016/j.celrep.2019.11.029 (2019).

46. Thomzig, A., Kratzel, C., Lenz, G., Krüger, D. \& Beekes, M. Widespread prpsc accumulation in muscles of hamsters orally infected with scrapie. EMBO Rep. 4, 530-533. https://doi.org/10.1038/sj.embor.embor827 (2003).

\section{Acknowledgements}

We are grateful to Helmut Kettenmann (Max Delbrück Center, Berlin, Germany) for sharing his protocol for murine primary glia cell isolation and to Maren Wendt for the technical training; to Julia Madela-Mönchinger (RKI) for her flow cytometry guidance; to Umberto Agrimi and Michele Di Bari (Istituto Superiore di Sanità, Rome, Italy) for kindly providing breeding pairs of bank voles; to Sabine Gilch and Hermann Schätzl (University of Calgary, Canada) for kindly providing the murine and bank vole-adapted scrapie strains; to the animal facility of RKI for their support and to Sophia Schwartz for technical assistance.

\section{Author contributions}

K.A.S. and J.H.W. conceived and performed the experiments and analysed the results. A.B. guided the flow cytometry experiment and analysed the data. K.A.S., J.H.W., A.T. and M.B. contributed to study conceptualisation and data interpretation. M.B. raised the funding. K.A.S. drafted the manuscript. All authors revised and approved the final manuscript.

\section{Funding}

Open Access funding enabled and organized by Projekt DEAL. KAS was financially supported by Alberta Prion Research Institute (Alberta, Canada, project number PEX12015) and RKI. JHW was financially supported by German Federal Ministry of Education and Research (funding initiative "Alternative methods to animal experiments", project number 031L0065).

\section{Competing interests}

The authors declare no competing interests.

\section{Additional information}

Supplementary Information The online version contains supplementary material available at https://doi.org/ 10.1038/s41598-022-06198-4.

Correspondence and requests for materials should be addressed to K.A.S.

Reprints and permissions information is available at www.nature.com/reprints.

Publisher's note Springer Nature remains neutral with regard to jurisdictional claims in published maps and institutional affiliations.

\footnotetext{
(c) (i) Open Access This article is licensed under a Creative Commons Attribution 4.0 International cc) License, which permits use, sharing, adaptation, distribution and reproduction in any medium or format, as long as you give appropriate credit to the original author(s) and the source, provide a link to the Creative Commons licence, and indicate if changes were made. The images or other third party material in this article are included in the article's Creative Commons licence, unless indicated otherwise in a credit line to the material. If material is not included in the article's Creative Commons licence and your intended use is not permitted by statutory regulation or exceeds the permitted use, you will need to obtain permission directly from the copyright holder. To view a copy of this licence, visit http://creativecommons.org/licenses/by/4.0/.
}

(C) The Author(s) 2022 